\title{
Sodium glucose co-transporter 2 inhibitors mediated ketogenesis in patients with metabolic syndrome: clear benefit or anticipated fear?
}

Dimitrios loannis Patoulias

First Department of Internal Medicine, General Hospital “Hippokration”, Thessaloniki, Greece

Submitted: 25 October 2018

Accepted: 2 February 2019

Arch Med Sci Atheroscler Dis 2019; 4: e13-e15

DOI: https://doi.org/10.5114/amsad.2019.83302

Copyright $\odot 2019$ Termedia \& Banach

Sodium glucose co-transporter-2 (SGLT-2 inhibitors) have gained significant ground in the field of therapeutics of type 2 diabetes mellitus (T2DM) [1]. Recently, Gonzalez-Ortiz et al. demonstrated that dapagliflozin, a sodium glucose co-transporter 2 (SGLT-2) inhibitor, is also effective in patients with metabolic syndrome (MetS), but without T2DM [2]. The researchers showed that dapagliflozin led to a significant improvement in the parameters of MetS, resulting in remission of MetS in $58.3 \%$ of all stratified participants [2]. This observation may reflect the multiple, pleiotropic effects of this novel drug class.

It is established that SGLT-2 inhibitors enhance ketogenesis via mediating the decrease in insulin secretion (due to glucosuria and subsequent decrease in blood glucose levels) and counter-regulatory increase in glucagon levels, while there is evidence of direct action of SGLT-2 inhibitors on pancreatic $\alpha$-cells [3]. Modest, but sustained ketone body reabsorption may also contribute to increased ketone body levels in subjects treated with SGLT-2 inhibitors. Ketogenesis is associated with a substantial increase in lipolysis rates, with shifting from glucose to free fatty acids as an energy substrate [3]. Gonzalez-Ortiz et al. demonstrated that dapagliflozin treatment in the corresponding arm resulted in a significant decrease in triglyceride levels and insulin secretion, which provides evidence on the background pathophysiologic mechanism [2].

The "hepatic fatty acid drainage hypothesis" and its implications in MetS have been formulated by Berge et al. [4]. Stimulation of ketogenesis and subsequent free fatty acid drainage from the liver can relieve fatty acid pressure on the adipose tissue and the skeletal muscle, improving insulin sensitivity and glucose uptake, along with a decrease in adipose tissue and ectopic fat accumulation [4]. Experimental data evince that impaired $\beta$-oxidation is closely related to severe insulin resistance and vice versa [5], while ketogenesis arises as a crucial regulator of glucose metabolism and fatty liver disease [6].

Despite the fact that there are insufficient data concerning the implications of pharmacologically induced ketogenesis in MetS, previous data support the application of nutritional ketosis in patients with MetS [7]. Utilization of free fatty acids and generated ketones as an alternative fuel substrate results in significant improvement in the major components of MetS. Although it is difficult to quantify and compare the keto-

\author{
Corresponding author: \\ Dimitrios loannis Patoulias \\ MD \\ First Department \\ of Internal Medicine \\ General Hospital \\ "Hippokration" \\ Thessaloniki, Greece \\ Phone: +30 6946900777 \\ E-mail: dipatoulias@gmail. \\ com
}


genic effects of a nutritional model to those mediated by a drug class, we must admit that there are certain similarities at the pathophysiologic level.

Additional data suggest that enhanced ketogenesis mediated by SGLT-2 inhibitors is associated with cardioprotection and renoprotection [8]. Previous data also support that ketogenesis is in fact a compensatory mechanism to hyperglycemia, correlated with cardio- and reno-protection, along with decreased incidence rates of all-cause mortality [9]. Based on the established association between MetS, all-cause mortality and cardiovascular mortality [10,11], it is deduced that SGLT-2 inhibitors provide a promising therapeutic perspective in patients with MetS, and thus, underlying pathophysiologic mechanisms should be highlighted.

It seems that there is a sensitive balance between ketogenesis and ketoacidosis, with the existence of several underlying pathophysiologic mechanisms, including the aforementioned, that could interpret the association between the administration of SGLT-2 inhibitors and the occurrence of diabetic ketoacidosis [12]. Physicians should apply extra caution when prescribing this novel class of drugs in patients with T2DM, although the true risk has yet to be elucidated in the real-world setting [13]. Data retrieved from the most recent meta-analysis of 8 randomized controlled trials involving 10,157 patients on an SGLT-2 inhibitor-based regimen depict negligible odds for ketoacidosis with the administration of this drug class [14].

However, according to the results obtained from the recently published meta-analysis of the three hallmark cardiovascular and renal outcome trials, namely the Empagliflozin Cardiovascular Outcome Event Trial in Type 2 Diabetes Mellitus Patients-Removing Excess Glucose (EMPA-REG OUTCOME) [15], the Canagliflozin Cardiovascular Assessment Study (CANVAS) [16] and the Dapagliflozin Effect on Cardiovascular Events-Thrombolysis in Myocardial Infarction 58 (DECLARE-TIMI 58) [17], administration of an SGLT-2 inhibitor in patients with T2DM increase more than twofold the risk of diabetic ketoacidosis, despite the quite low event rates [18]. Cohort studies will shed light on those findings [13].

In conclusion, SGLT-2 inhibitors offer a great perspective for the treatment of subjects with MetS, with or without concomitant T2DM; thus, we should focus on the beneficial ketogenic effects of this drug class. Further clinical trials, similar to that conducted by Gonzalez-Ortiz et al., but with quantification of the ketogenic effect of the administered SGLT-2 inhibitor, along with precise correlation estimates with MetS components, mainly body weight, glycemic control, lipid profile and blood pressure, will elucidate this reasonable hypothesis.

\section{Conflict of interest}

The author declares no conflict of interest.

\section{References}

1. Davies MJ, D'Alessio DA, Fradkin J, et al. Management of hyperglycemia in type 2 Diabetes, 2018. A Consensus Report by the American Diabetes Association (ADA) and the European Association for the Study of Diabetes (EASD). Diabetes Care 2018; 41: 2669-701.

2. González-Ortiz M, Méndez-Del Villar M, Martínez-Abundis E, Ramírez-Rodriguez AM. Effect of dapagliflozin administration on metabolic syndrome, insulin sensitivity, and insulin secretion. Minerva Endocrinol 2018; 43: 229-35.

3. Qiu H, Novikov A, Vallon V. Ketosis and diabetic ketoacidosis in response to SGLT2 inhibitors: basic mechanisms and therapeutic perspectives. Diabetes Metab Res Rev 2017; 33. doi: 10.1002/dmrr.2886. Epub 2017 Feb 23.

4. Berge RK, Tronstad KJ, Berge K, et al. The metabolic syndrome and the hepatic fatty acid drainage hypothesis. Biochimie 2005; 87: 15-20.

5. Satapati S, Sunny NE, Kucejova B, et al. Elevated TCA cycle function in the pathology of diet-induced hepatic insulin resistance and fatty liver. J Lipid Res 2012; 53: 1080-92.

6. Cotter DG, Ercal B, Huang X, et al. Ketogenesis prevents diet-induced fatty liver injury and hyperglycemia. J Clin Invest 2014; 124: 5175-90.

7. Gershuni VM, Yan SL, Medici V. Nutritional ketosis for weight management and reversal of metabolic syndrome. Curr Nutr Rep 2018; 7: 97-106.

8. Ferrannini E, Mark M, Mayoux E. CV Protection in the EMPA-REG OUTCOME trial: a "thrifty substrate" hypothesis. Diabetes Care 2016; 39: 1108-14.

9. Kruljac I, Ćaćić M, Ćaćić P, et al. Diabetic ketosis during hyperglycemic crisis is associated with decreased allcause mortality in patients with type 2 diabetes mellitus. Endocrine 2017; 55: 139-43.

10. Ju SY, Lee JY, Kim DH. Association of metabolic syndrome and its components with all-cause and cardiovascular mortality in the elderly: a meta-analysis of prospective cohort studies. Medicine (Baltimore) 2017; 96: e8491.

11. Tie HT, Shi R, Li ZH, Zhang M, Zhang C, Wu QC. Risk of major adverse cardiovascular events in patients with metabolic syndrome after revascularization: a metaanalysis of eighteen cohorts with 18,457 patients. Metabolism 2015; 64: 1224-34.

12. Taylor SI, Blau JE, Rother KI. SGLT2 inhibitors may predispose to ketoacidosis. J Clin Endocrinol Metab 2015; 100: 2849-52.

13. Fadini GP, Bonora BM, Avogaro A. SGLT2 inhibitors and diabetic ketoacidosis: data from the FDA Adverse Event Reporting System. Diabetologia 2017; 60: 1385-9.

14. Monami M, Nreu B, Zannoni S, Lualdi C, Mannucci E. Effects of SGLT-2 inhibitors on diabetic ketoacidosis: a meta-analysis of randomised controlled trials. Diabetes Res Clin Pract 2017; 130: 53-60.

15. Zinman B, Wanner C, Lachin JM, et al. Empagliflozin, cardiovascular outcomes, and mortality in type 2 diabetes. N Engl J Med 2015; 373: 2117-28. 
16. Neal B, Perkovic V, Mahaffey KW, et al. Canagliflozin and cardiovascular and renal events in type 2 diabetes. N Engl J Med 2017; 377: 644-57.

17. Wiviott SD, Raz I, Bonaca MP, et al. Dapagliflozin and cardiovascular outcomes in type 2 diabetes. N Engl J Med 2019; 380: 347-57.

18. Zelniker TA, Wiviott SD, Raz I, et al. SGLT2 inhibitors for primary and secondary prevention of cardiovascular and renal outcomes in type 2 diabetes: a systematic review and meta-analysis of cardiovascular outcome trials. Lancet 2019; 393: 31-9. 